\title{
Compatibilidade de fungos entomopatogênicos com agroquímicos*
}

\section{Compatibility between entomopathogenic fungi and agrochemicals}

\author{
Renato Cassol de Oliveira ${ }^{1}$; Pedro Manuel Oliveira Janeiro Neves ${ }^{2}$; \\ Élio Cesar Guzzo ${ }^{3}$; Viviane Sandra Alves ${ }^{3}$
}

\begin{abstract}
Resumo
A compatibilidade das formulações Citrex SC (clorfenapyr), Kendo SC (fenpyroximate), Parsec CE (amitraz), Rufast 50SC (acrinathrin), Sanmite CE (pyridaben), Vertimec 18 CE (abamectim), Savey PM (hexythioazox) e Partner SC (fenbutatin), foi avaliada em duas concentrações - concentração média: $\mathrm{CM}(1 \mathrm{x})$ e a metade da $\mathrm{CM}(0,5 \mathrm{x})$ sobre a germinação, crescimento vegetativo e conidiogênese dos fungos entomopatogênicos Beauveria bassiana, Metarhizium anisopliae e Paecilomyces fumosoroseus. As formulações Rufast 50SC (acrinathrin), Vertimec 18CE (abamectin) e Savey PM (hexythioazox) foram seletivas aos fungos, com maior potencial para serem utilizados em programas de manejo integrado de pragas, de forma associada com o patógeno ou com o objetivo de preservar o potencial de inóculo destes fungos presentes nos agroecossistemas.

Palavras-chave: Grupos químicos, seletividade, fungitóxico.
\end{abstract}

\begin{abstract}
Citrex SC (clorfenapyr), Kendo SC (fenpyroximate), Parsec CE (amitraz), Rufast 50SC (acrinathrin), Sanmite CE (pyridaben), Vertimec 18 CE (abamectim), Savey PM (hexythioazox) e Partner SC (fenbutatin) formulation compatibility was estimated in two concentration - medium concentration: $\mathrm{MC}(1 \mathrm{x})$ and a half $\mathrm{MC}(0,5 \mathrm{x})$ on germination, vegetative grow and condiogenes of the enthomopathogenic fungi Beauveria bassiana, Metarhizium anisopliae and Paecilomyces fumosoroseus. Rufast 50SC (acrinathrin), Vertimec 18CE (abamectin) e Savey PM (hexythioazox) formulation were selective to the fungi, with potential to be used in integrate pest management (IPM), associated with the pathogen or with objective of preserving the potential of inoculum potential of this fungi present in the agroecosystems.

Key word: Chemical groups, selectivity, fungi toxic.
\end{abstract}

\section{Introdução}

Existem evidências de que produtos químicos utilizados na proteção das culturas podem ter efeitos antagônicos, nulos ou sinérgicos sobre a atividade inseticida/acaricida dos entomopatógenos presentes no agroecossistema (BENZ, 1987). Assim, conhecer a compatibilidade destes produtos sobre as diversas fases de desenvolvimento dos fungos entomopatogênicos é essencial em programas de Manejo Integrado de Pragas (MIP), onde os entomopatógenos são importantes agentes naturais de controle de pragas. A preservação da viabilidade dos conídios é de extrema importância, pois estas estruturas são as responsáveis pela ocorrência da

\footnotetext{
1 Depto. de Ciências Biológicas - UNIPAR, C.P. 4515, CEP: 85801-470, Cascavel, PR. E-mail: renato_cassol@hotmail.com.

2 Depto. de Agronomia, CCA/ UEL. Londrina, PR. C.P. 6001. CEP: 86051-990. E-mail: pmojneve@uel.br.

3 Graduandos do Curso de Ciências Biológicas - UNIOESTE. Cascavel, PR.

* Trabalho desenvolvido no Laboratório de Entomologia do Depto. de Agronomia CCA - UEL.
} 
doença nas populações de insetos (DUARTE; MENENDEZ; TRIGUEIRO, 1992; TODOROVA et al., 1998). É também importante a sua preservação, quando o fungo (conídios) é aplicado a campo, de modo inundativo associado ou não a produtos fitossanitários (ANDERSON; ROBERTS, 1983; NEVES et al., 2001).

Assim, avaliou-se a compatibilidade (seletividade), in vitro, de algumas formulações sobre a germinação, crescimento vegetativo e conidiogênese dos fungos entomopatogênicos Beauveria bassiana, Metarhizium anisopliae e Paecilomyces fumosoroseus.

\section{Material e Métodos}

Fungos Entomopatogênicos. Utilizou-se o fungo $B$. bassiana (isolado 447) e M. anisopliae (isolado E9) Esalq - USP e P. fumosoroseus (isolado CG120) EMBRAPA - CENARGEN, Brasília, mantidos no Banco de Patógenos do Laboratório de Controle Microbiano do Departamento de Agronomia da UEL. Os isolados armazenados a $-4^{\circ} \mathrm{C}$, foram multiplicados em meio de cultura B.D.A. $\left(25 \pm 1{ }^{\circ} \mathrm{C} ; 12 \mathrm{~h}\right.$ fotofase). Os conídios produzidos foram utilizados nos testes de germinação, crescimento vegetativo e conidiogênese.

Produtos fitossanitários. Informações sobre o ingrediente ativo, nome comercial, formulação, grupo químico, bem como as concentrações (ppm), são apresentadas na Tabela 1. As formulações foram avaliadas em duas concentrações: concentração média $(C M=1 x)$ e metade da CM $(0,5 x)$. Para determinação da CM calculou-se a média aritmética utilizando-se as várias concentrações recomendadas pelos fabricantes para aplicação a campo, em 100 litros de água/ha, nas diferentes culturas.
Tabela 1 - Agroquímicos utilizadas no teste de compatibilidade com fungos entomopatogênicos.

\begin{tabular}{llllr}
\hline $\begin{array}{l}\text { Nome } \\
\text { comercial }\end{array}$ & $\begin{array}{c}\text { Ingrediente } \\
\text { ativo }\end{array}$ & Formulação & Grupo Químico & ppm \\
\hline Citrex & Clorfenapyr & SC & Pyrrol & 470 \\
Kendo & Fenpyroximate & SC & Pyrazol & 1000 \\
Parsec & Amitraz & CE & Formamidinas & 1750 \\
Rufast 50 & Acrinathrin & SC & Ester Nor-pirétrico & 100 \\
Sanmite & Pyridaben & CE & Piridazinonas & 625 \\
Vertimec & Abamectin & CE & Avermectinas & 650 \\
Savey & Hexythioazox & PM & Carboxamida & 30 \\
Partner & Fenbutatin & SC & Organo estânico & 600 \\
\hline
\end{tabular}

* SC (suspensão concentrada), CE (concentrado emulsionável), PM (pó molhável), WG (wettable granulated).

**Concentração Média para aplicação em 100 L/ha.

Para os testes de germinação, as formulações, nas concentrações preestabelecidas, foram misturadas em água destilada estéril contendo Tween $20(0,02 \%)$ e $1 \mathrm{~mL}$ da suspensão de conídios, padronizada em $1 \times 10^{6}$ conídios $/ \mathrm{mL}$. Decorridos 60 minutos, alíquotas de $0,5 \mathrm{~mL}$ foram espalhadas, com o auxílio da alça de Drigalsky, em placas de Petri contendo uma fina camada de meio de cultura ágar-água. No tratamento controle utilizou-se suspensão do fungo em água destilada estéril + Tween $20(0,02 \%)$. Para cada tratamento (concentrações preestabelecidas de cada formulação) foram feitas quatro repetições (placas). Após 20 horas avaliou-se a viabilidade, dividindose as placas em 4 quadrantes quantificando-se os geminados e os não germinados, aproximadamente 100 conídios/quadrante, sob de microscópio óptico. Os valores obtidos foram utilizados para estabelecimento da porcentagem de germinação.

Nos testes de crescimento vegetativo e conidiogênese, o meio B.D.A. foi autoclavado a $120^{\circ} \mathrm{C}$ por $20 \mathrm{~min}$., resfriado a $40 \pm 5^{\circ} \mathrm{C}$ acrescentando-se posteriormente as formulações, nas concentrações preestabelecidas, juntamente com o antibiótico estreptomicina $(0,5 \mathrm{~g} / \mathrm{L})$. Em placas de Petri (9 $\mathrm{cm} \varnothing$ ), foram vertidos $\pm 20 \mathrm{~mL}$ da mistura. No tratamento controle utilizou-se B.D.A. + antibiótico. Para cada tratamento (fungo/concentração) foram feitas 6 repetições (placas), nas quais inocularam-se os conídios em três pontos eqüidistantes. Após 8 dias, o diâmetro das colônias foi determinado com auxílio de paquímetro, fazendo-se 3 medidas por colônia, 
utilizando-se estes valores para o cálculo da área de cada colônia.

Posteriormente, retirou-se, com auxílio de um vazador, um disco de $15 \mathrm{~mm} \varnothing$, do centro de cada colônia, num total de 10 colônias/tratamento, para avaliação da produção de conídios. Os discos foram suspensos, separadamente, em $10 \mathrm{~mL}$ de água estéril + Tween20 $(0,02 \%)$ em tubos de vidro de fundo chato $(8,40 \mathrm{~cm}$ altura por $2,30 \mathrm{~cm} \varnothing)$, agitados até o completo desprendimento dos conídios, com subsequentes diluições e posterior quantificação em câmara de Neubauer.

O delineamento estatístico foi o inteiramente casualizado. Os dados obtidos foram analisados utilizando-se o PROC ANOVA e o programa estatístico SAS (Statistical Analytical Systems) (SAS Institute, 1998). Todas as placas, dos dois experimentos, foram mantidas em B.O.D. a $25 \pm 1^{\circ} \mathrm{C}$ e fotofase de $12 \mathrm{~h}$.

\section{Resultados e Discussão}

\section{Germinação}

Para B. bassiana, as formulações Rufast e Vertimec $(0,5 \mathrm{x}$ e $1 \mathrm{x})$ Savey e Parsec $(0,5 \mathrm{x})$, causaram reduções pouco expressivas na germinação, não diferindo estatisticamente em relação à testemunha. Já os tratamentos com as formulações Kendo, Sanmite, Citrex (0,5x e 1x) e Savey, Parsec (1x) causaram reduções entre 17 e $40 \%$. A formulação Partner, nas duas concentrações, afetou drasticamente a germinação de $B$. bassiana, com redução de até $85 \%$ (Tabela 2).

Para M. anisopliae, apenas o tratamento com Vertimec $(0,5 \mathrm{x})$ não diferiu estatisticamente da testemunha. Por outro lado, as formulações Partner $(0,5 \mathrm{x}$ e $1 \mathrm{x})$ causaram reduções na germinação $>85 \%$, diferindo significativamente da testemunha. As demais formulações testadas também diferiram da testemunha com reduções que variaram de $15 \mathrm{e}$ $45 \%$ (Tabela 3 ).

Tabela 2 - Percentagem de germinação de conídios (média $\pm \mathrm{DP}$ ), crescimento vegetativo (média $\pm \mathrm{DP}$ ) e conidiogênese (média $\pm \mathrm{DP}$ ) de Beauveria bassiana (isolado 447 ) a $25 \pm 1^{\circ} \mathrm{C}$ e fotofase $12 \mathrm{~h}$.

\begin{tabular}{|c|c|c|c|c|c|c|c|}
\hline \multirow[b]{2}{*}{ Tratamentos } & \multirow[b]{2}{*}{ Doses } & \multicolumn{2}{|c|}{ Germinação $(\%)$} & \multicolumn{2}{|c|}{ Crescimento vegetativo (mm) } & \multicolumn{2}{|c|}{ Conidiogênese (conídios $/ \mathrm{mL}^{4}$ ) } \\
\hline & & Médias $\pm \mathrm{DP}^{1}$ & \% Redução & Médias $\pm \mathrm{DP}^{2}$ & \% Redução & Médias $\pm \mathrm{DP}^{3}$ & \% Redução \\
\hline Testemunha & 0 & $91.59 \pm 0.00 \mathrm{~A}$ & 0.00 & $34.44 \pm 0.00 \mathrm{~A}$ & 0.00 & $96.37 \pm 0.00 \mathrm{C}$ & 0.00 \\
\hline Citrex SC & $0.5 \mathrm{x}$ & $59.13 \pm 5.98 \mathrm{E}$ & 35.44 & na & & na & \\
\hline Citrex SC & $1 \mathrm{x}$ & $68.71 \pm 10.37 \mathrm{DEC}$ & 24.98 & na & & na & \\
\hline Kendo SC & $0.5 x$ & $75.97 \pm 9.78 \mathrm{BDC}$ & 17.05 & na & & na & \\
\hline Kendo SC & $1 x$ & $67.04 \pm 6.29 \mathrm{DEC}$ & 26.80 & na & & na & \\
\hline Parsec CE & $0.5 x$ & $80.30 \pm 6.31 \mathrm{BAC}$ & 12.33 & $13.05 \pm 0.53 \mathrm{C}$ & 62.11 & $9.82 \pm 5.86 \mathrm{~F}$ & 89.81 \\
\hline Parsec CE & $1 \mathrm{x}$ & $63.77 \pm 6.63 \mathrm{DE}$ & 30.37 & $15.22 \pm 0.30 \mathrm{C}$ & 55.81 & $28.10 \pm 11.39 \mathrm{EF}$ & 70.84 \\
\hline Partner SC & $0.5 x$ & $13.73 \pm 3.12 \mathrm{~F}$ & 85.01 & na & & na & \\
\hline Partner SC & $1 \mathrm{x}$ & $14.40 \pm 5.57 \mathrm{~F}$ & 84.28 & na & & na & \\
\hline Rufast $50 \mathrm{SC}$ & $0.5 \mathrm{x}$ & $79.19 \pm 5.85 \mathrm{BAC}$ & 13.54 & $30.99 \pm 5.76 \mathrm{AB}$ & 10.02 & $124.62 \pm 17.80 \mathrm{~B}$ & +29.31 \\
\hline Rufast $50 \mathrm{SC}$ & $1 \mathrm{x}$ & $76.90 \pm 3.28 \mathrm{BDAC}$ & 16.04 & $34.16 \pm 1.32 \mathrm{~A}$ & 0.81 & $155.62 \pm 21.36 \mathrm{~A}$ & +61.48 \\
\hline Sanmite CE & $0.5 \mathrm{x}$ & $66.12 \pm 5.81 \mathrm{DEC}$ & 27.81 & $10.77 \pm 5.58 \mathrm{C}$ & 68.73 & $13.21 \pm 14.97 \mathrm{~F}$ & 86.29 \\
\hline Sanmite CE & $1 \mathrm{x}$ & $55.86 \pm 5.62 \mathrm{E}$ & 39.01 & $15.05 \pm 2.81 \mathrm{C}$ & 56.30 & $32.73 \pm 30.19 \mathrm{EF}$ & 66.04 \\
\hline Savey PM & $1 \mathrm{x}$ & $74.63 \pm 5.87 \mathrm{BDC}$ & 18.52 & $33.11 \pm 1.36 \mathrm{~A}$ & 3.86 & $105.14 \pm 71.77 \mathrm{BC}$ & +9.10 \\
\hline Vertimec CE & $0.5 \mathrm{x}$ & $80.53 \pm 3.70 \mathrm{BAC}$ & 12.08 & $26.68 \pm 0.32 \mathrm{~B}$ & 22.53 & $42.66 \pm 3.93 \mathrm{DE}$ & 55.73 \\
\hline Vertimec CE & $1 \mathrm{x}$ & $84.24 \pm 3.68 \mathrm{BA}$ & 8.02 & $29.46 \pm 0.64 \mathrm{AB}$ & 14.46 & $88.01 \pm 11.29 \mathrm{C}$ & 8.67 \\
\hline
\end{tabular}

Médias seguidas pela mesma letra, na coluna, não diferem entre si pelo teste de Tukey $(\mathrm{P}<0,05)$, ${ }^{1} \mathrm{n}=4 ;{ }^{2} \mathrm{n}=10 ;{ }^{3} \mathrm{n}=8 ;{ }^{4} \times 10^{5}$ conídios/mL; na - não avaliado; + superior ao tratamento controle 
Tabela 3 - Percentagem de germinação de conídios (média $\pm \mathrm{DP}$ ), crescimento vegetativo (média $\pm \mathrm{DP}$ ) e conidiogênese (média $\pm \mathrm{DP}$ ) de Metarhizium anisopliae (isolado E9) a $25 \pm 1{ }^{\circ} \mathrm{C}$ e fotofase $12 \mathrm{~h}$.

\begin{tabular}{|c|c|c|c|c|c|c|c|}
\hline \multirow[b]{2}{*}{ Tratamento } & \multirow[b]{2}{*}{ Doses } & \multicolumn{2}{|c|}{ Germinação (\%) } & \multicolumn{2}{|c|}{ Crescimento vegetativo $(\mathrm{mm})$} & \multicolumn{2}{|c|}{ Conidiogênese (conídios $/ \mathrm{mL}^{4}$ ) } \\
\hline & & Médias $\pm \mathrm{DP}^{1}$ & \% Redução & Médias $\pm \mathrm{DP}^{2}$ & \% Redução & Médias $\pm \mathrm{DP}^{3}$ & \% Redução \\
\hline Testemunha & 0 & $90.99 \pm \mathrm{A}$ & 0.00 & $26.59 \pm 0.00 \mathrm{AB}$ & 0.00 & $130.00 \pm 0.00 \mathrm{~A}$ & 0.00 \\
\hline Citrex SC & $0.5 \mathrm{x}$ & $62.15 \pm 7.99 \mathrm{EFD}$ & 31.70 & na & & na & \\
\hline Citrex SC & $1 \mathrm{x}$ & $50.65 \pm 4.95 \mathrm{~F}$ & 44.33 & na & & na & \\
\hline Kendo SC & $0.5 \mathrm{x}$ & $69.43 \pm 6.20 \mathrm{CD}$ & 23.69 & na & & na & \\
\hline Kendo SC & $1 \mathrm{x}$ & $54.82 \pm 4.73 \mathrm{EF}$ & 39.75 & na & & na & \\
\hline Parsec CE & $0.5 \mathrm{x}$ & $70.75 \pm 5.95 \mathrm{CD}$ & 22.24 & $10.05 \pm 1.09 \mathrm{C}$ & 62.20 & $7.96 \pm 2.79 \mathrm{~B}$ & 93.88 \\
\hline Parsec CE & $1 \mathrm{x}$ & $65.21 \pm 5.43 \mathrm{ECD}$ & 28.33 & $8.16 \pm 2.06 \mathrm{C}$ & 69.31 & $15.97 \pm 1.88 \mathrm{~B}$ & 87.72 \\
\hline Partner SC & $0.5 \mathrm{x}$ & $12.43 \pm 3.61 \mathrm{G}$ & 86.34 & na & & na & \\
\hline Partner SC & $1 \mathrm{x}$ & $6.39 \pm 1.70 \mathrm{G}$ & 92.98 & na & & na & \\
\hline Rufast $50 \mathrm{SC}$ & $0.5 \mathrm{x}$ & $73.69 \pm 2.21 \mathrm{BCD}$ & 19.01 & $29.77 \pm 0.25 \mathrm{~A}$ & +11.96 & $113.25 \pm 8.50 \mathrm{~A}$ & 12.88 \\
\hline Rufast $50 \mathrm{SC}$ & $1 \mathrm{x}$ & $67.22 \pm 4.74 \mathrm{ECD}$ & 26.12 & $28.14 \pm 0.95 \mathrm{~A}$ & +5.83 & $152.62 \pm 7.50 \mathrm{~A}$ & +17.40 \\
\hline Sanmite CE & $0.5 \mathrm{x}$ & $70.76 \pm 4.14 \mathrm{CD}$ & 22.23 & $9.24 \pm 1.04 \mathrm{C}$ & 65.25 & $5.02 \pm 1.92 \mathrm{~B}$ & 96.14 \\
\hline Sanmite CE & $1 \mathrm{x}$ & $68.32 \pm 4.74 \mathrm{CD}$ & 24.91 & $7.16 \pm 4.17 \mathrm{C}$ & 73.07 & $6.63 \pm 1.65 \mathrm{~B}$ & 94.90 \\
\hline Savey PM & $0.5 \mathrm{x}$ & $76.39 \pm 2.78 \mathrm{BC}$ & 16.05 & $29.33 \pm 0.45^{\mathrm{A}}$ & +10.30 & $121.25 \pm 5.17 \mathrm{~A}$ & 6.73 \\
\hline Savey PM & $1 \mathrm{x}$ & $74.59 \pm 8.16 \mathrm{BCD}$ & 18.02 & $29.27 \pm 0.99^{\mathrm{A}}$ & +10.08 & $138.37 \pm 4.20 \mathrm{~A}$ & +6.44 \\
\hline Vertimec CE & $0.5 x$ & $83.85 \pm 4.57 \mathrm{BA}$ & 7.85 & $21.85 \pm 3.20 \mathrm{~B}$ & 17.83 & $116.25 \pm 8.50 \mathrm{~A}$ & 10.58 \\
\hline Vertimec CE & $1 \mathrm{x}$ & $67.66 \pm 3.61 \mathrm{CD}$ & 25.64 & $22.16 \pm 3.88 \mathrm{~B}$ & 16.66 & $143.25 \pm 9.80 \mathrm{~A}$ & +10.19 \\
\hline
\end{tabular}

Médias seguidas pela mesma letra, na coluna, não diferem entre si pelo teste de Tukey $(\mathrm{P}<0,05)$, ${ }^{1} \mathrm{n}=4 ;{ }^{2} \mathrm{n}=10 ;{ }^{3} \mathrm{n}=8 ;{ }^{4} \times 10^{5}$ conídios/mL; na - não avaliado; + superior ao tratamento controle

Para $P$. fumosoroseus, as formulações Citrex, Vertimec e Sanmite $(0,5 \mathrm{x}$ e1x) causaram reduções menores que $19 \%$, não diferindo estatisticamente da testemunha. Rufast, Parsec e Kendo, nas duas concentrações, reduziram a germinação entre 20 e $60 \%$, em relação à testemunha, diferindo estatisticamente desta. Já as formulações partner $(0,5 \mathrm{x}$ e $1 \mathrm{x})$ afetaram drasticamente a germinação, com redução $>70 \%$ (Tabela 4).

Ghimi e Kimati (2000), relatam que para fungos fitopatogênicos, as formulações do grupo dos organofosforados agem inibindo a enzima que converte fofatidiletanolamina para fosfatidilcolina, inibindo também a síntese de quitina e interferindo na produção da parede celular. Provavelmente, este seja o mesmo mecanismo que provocou um efeito drástico na germinação dos conídios para os três fungos, quando em contato com a formulação Partner (organoestânico).

Já a formulação Vertimec não apresentou atividade antifúngica significativa, tal como discutido por Halley et al. (1993) que avaliaram o efeito das avermetinas.

\section{Crescimento vegetativo e conidiogênese}

Nesta etapa, somente foram testadas as formulações que causaram reduções na germinação inferiores a 40\%, em relação aos três fungos entomopatogênicos.

Assim, para o crescimento vegetativo de $B$. bassiana, Savey, Rufast (0,5x e 1x) e Vertimec (0,5x) causaram reduções menores do que $15 \%$, não diferindo da testemunha. Vertimec $(1 \mathrm{x})$ reduziu em $22,5 \%$ o crescimento vegetativo e as formulações Parsec e Sanmite (0,5x e 1x), diferiram significativamente da testemunha, com reduções do crescimento vegetativo maiores que $55 \%$ (Tabela 2 ).

$\mathrm{O}$ crescimento vegetativo de $M$. anisopliae nos tratamentos contendo as formulações Vertimec, Savey e Rufast $(0,5 \mathrm{x}$ e $1 \mathrm{x})$, não diferiram estatisticamente da testemunha. Todavia, novamente as formulações Parsec e Sanmite $(0,5 x$ e 1x), diferiram significativamente da testemunha, com percentagem de redução do crescimento vegetativa superiores a $62 \%$ (Tabela 3 ). 
Para P. fumosoroseus as formulações Rufast $(0,5 \mathrm{x}$ e 1x) e Savey (1x) não provocaram níveis de inibição estatisticamente diferentes da testemunha. Já Savey $(0,5 x)$ e Vertimec $(0,5 x$ e $1 x)$ promoveram inibições estatisticamente diferentes da testemunha. As formulações Parsec e Sanmite (0,5x e 1x), também causaram reduções do crescimento vegetativo superiores a $42 \%$, em relação à testemunha (Tabela 4).

Tabela 4 - Percentagem de germinação de conídios (média $\pm \mathrm{DP}$ ), crescimento vegetativo (média $\pm \mathrm{DP}$ ) e conidiogênese (média $\pm \mathrm{DP}$ ) de Paecilomyces fumosoroseus (isolado CB120) a $25 \pm 1^{\circ} \mathrm{C}$ e fotofase $12 \mathrm{~h}$.

\begin{tabular}{|c|c|c|c|c|c|c|c|}
\hline \multirow[b]{2}{*}{ Tratamentos } & \multirow[b]{2}{*}{ Doses } & \multicolumn{2}{|c|}{ Germinação (\%) } & \multicolumn{2}{|c|}{ Crescimento vegetativo (mm) } & \multicolumn{2}{|c|}{ Conidiogênese (conídios $/ \mathrm{mL}^{4}$ ) } \\
\hline & & Médias $\pm \mathrm{DP}^{1}$ & \% Redução & Médias $\pm \mathrm{DP}^{2}$ & \% Redução & Médias $\pm \mathrm{DP}^{3}$ & \% Redução \\
\hline Testemunha & 0 & $86.96 \pm 0.00 \mathrm{~A}$ & 0.00 & $31.99 \pm 0.00 \mathrm{~A}$ & 0.00 & $137.49 \pm 0.00 \mathrm{BA}$ & 0.00 \\
\hline Citrex SC & $0.5 \mathrm{x}$ & $73.20 \pm 6.13 \mathrm{ABC}$ & 15.82 & na & & na & \\
\hline Citrex SC & $1 \mathrm{x}$ & $70.88 \pm 11.58 \mathrm{ABCD}$ & 18.49 & na & & na & \\
\hline Kendo SC & $0.5 \mathrm{x}$ & $53.98 \pm 10.1 \mathrm{EF}$ & 37.93 & na & & na & \\
\hline Kendo SC & $1 \mathrm{x}$ & $34.90 \pm 3.71 \mathrm{GH}$ & 59.87 & na & & na & \\
\hline Parsec CE & $0.5 \mathrm{x}$ & $55.88 \pm 6.28 \mathrm{DEF}$ & 35.74 & $17 \pm 00.63 \mathrm{D}$ & 46.86 & $17.70 \pm 14.53 \mathrm{D}$ & 87.13 \\
\hline Parsec CE & $1 \mathrm{x}$ & $49.35 \pm 6.43 \mathrm{FG}$ & 43.25 & $18.33 \pm 0.51 \mathrm{D}$ & 42.70 & $40.42 \pm 33.00 \mathrm{DC}$ & 70.60 \\
\hline Partner SC & $0.5 \mathrm{x}$ & $25.24 \pm 9.8 \mathrm{HI}$ & 70.98 & na & & na & \\
\hline Partner SC & $1 \mathrm{x}$ & $16.52 \pm 1.18 \mathrm{I}$ & 81.00 & na & & na & \\
\hline Rufast $50 \mathrm{SC}$ & $0.5 \mathrm{x}$ & $60.72 \pm 4.86 \mathrm{CDEF}$ & 30.17 & $30.66 \pm 1.17 \mathrm{AB}$ & 4.16 & $125.12 \pm 64.78 \mathrm{BA}$ & 9.00 \\
\hline Rufast $50 \mathrm{SC}$ & $1 \mathrm{x}$ & $53.82 \pm 4.2 \mathrm{EF}$ & 38.11 & $31.61 \pm 1.59 \mathrm{~A}$ & 1.19 & $150.75 \pm 16.00 \mathrm{~A}$ & +9.64 \\
\hline Sanmite CE & $0.5 \mathrm{x}$ & $77.70 \pm 6.79 \mathrm{AB}$ & 10.65 & $9.94 \pm 2.10 \mathrm{~F}$ & 68.93 & $8.58 \pm 44.00 \mathrm{D}$ & 93.76 \\
\hline Sanmite CE & $1 \mathrm{x}$ & $79.46 \pm 5.32 \mathrm{AB}$ & 8.62 & $14.29 \pm 1.00 \mathrm{E}$ & 55.33 & $26.45 \pm 7.33 \mathrm{D}$ & 80.76 \\
\hline Savey PM & $0.5 \mathrm{x}$ & $50.82 \pm 8.48 \mathrm{FG}$ & 41.56 & $30.38 \pm 0.99 \mathrm{AB}$ & 5.03 & $87.47 \pm 47.00 \mathrm{BC}$ & 36.38 \\
\hline Savey PM & $1 \mathrm{x}$ & $70.18 \pm 3.95 \mathrm{BCDE}$ & 19.30 & $28.94 \pm 0.82 \mathrm{~B}$ & 9.53 & $135.88 \pm 61.72 \mathrm{BA}$ & 1.17 \\
\hline Vertimec CE & $0.5 \mathrm{x}$ & $86.12 \pm 2.83 \mathrm{BA}$ & 0.97 & $26.27 \pm 1.20 \mathrm{C}$ & 17.88 & $94.25 \pm 61.94 \mathrm{BC}$ & 31.45 \\
\hline Vertimec CE & $1 \mathrm{x}$ & $83.81 \pm 2.85 \mathrm{BA}$ & 3.62 & $28.96 \pm 1.18 \mathrm{~B}$ & 9.47 & $178.37 \pm 10.96 \mathrm{~A}$ & +29.73 \\
\hline
\end{tabular}

Médias seguidas pela mesma letra, na coluna, não diferem entre si pelo teste de Tukey $(\mathrm{P}<0,05)$, ${ }^{1} \mathrm{n}=4 ;{ }^{2} \mathrm{n}=10 ;{ }^{3} \mathrm{n}=8 ;{ }^{4} \times 10^{5}$ conídios/mL; na - não avaliado; + superior ao tratamento controle

Em relação à influência das formulações sobre a conidiogênese, verifica-se que $B$. bassiana, teve um incremento superior a $60 \%$ na produção de conídios, nos tratamentos contento Rufast $(0,5 \mathrm{x}$ e $1 x)$, em relação à testemunha. Já as formulações Vertimec e Savey $(0,5 x)$ não promoveram diferenças significativas na conidiogênese em relação à testemunha. Nos demais tratamentos a produção de conídios foi significativamente afetada, com reduções de até 90\% (Tabela 2).

Para M. anisopliae e P. fumosoroseus, verificou-se uma redução significativa na esporulação nos tratamentos contendo as formulações Parsec e Sanmite $(0,5 x$ e $1 x)$.
A variação na inibição do crescimento vegetativo de B. bassiana, M. anisopliae e P. fumosoroseus, deve-se provavelmente ao mecanismo de mobilização dos nutrientes que cada fungo apresenta. Ghimi e Kimati (2000) ressaltam que para fungos fitopatogênicos, os produtos antifúngicos agem inibindo o acúmulo e incorporação de carboidratos na parede das hifas ou ainda inibindo a biossíntese de melanina.

Com a metabolização, pelos fungos, do meio contendo a formulação, pode ocorrer a liberação de resíduos tóxicos que se acumulam contra gradiente de concentração, bloqueiam as vias de compostos importantes para o funcionamento e cresci- 
mento do fungo. O fato de ocorrer germinação e crescimento vegetativo, se deve provavelmente ao lento processo de acúmulo do produto em níveis letais e consequentemente a inibição de vários processos vitais.

Em relação ao aumento na produção de conídios dos fungos $B$. bassiana, $M$. anisopliae e $P$. fumosoroseus, na presença de algumas formulações, Moino Jr. e Alves (1998) sugerem duas possíveis explicações para estes resultados:

a) os fungos, num mecanismo fisiológico de resistência, metabolizam compostos tóxicos que podem ser utilizados como nutrientes; e

b) em meio tóxico realizam um elevado esforço reprodutivo, aumentando a produção de conídios.

Porém, a inexistência de bibliografia com estudos de compatibilidade destas formulações com fungos entomopatogênicos, bem como a falta de uma metodologia de padronização para este tipo de teste, dificulta o aprofundamento da discussão.

Entretanto, com a metodologia utilizada, foi possível evidenciar as diferenças de compatibilidade das formulações sobre todas as fases de desenvolvimento dos fungos entomopatogênicos. Porém, estudos de campo precisam ser conduzidos para avaliar a interação e o efeito das formulações com os fungos entomopatogênicos, bem como os benefícios do uso associado destes, sobre as pragas.

\section{Conclusões}

Os produtos Rufast 50SC (acrinathrin), Vertimec 18CE (abamectin) e Savey PM (hexythioazox) são compatíveis/seletivos aos fungos B. bassiana, $M$. anisopliae e $P$. fumosoroseus e podem ser recomendados para uso em programas de Manejo Integrado de Pragas (MIP) onde os patógenos sejam importantes controladores de uma população de insetos e de ácaros.

\section{Agradecimentos}

A professora Dr. Inês Fonseca pelo auxílio na análise estatística e a CAPES pela bolsa de estudos.

\section{Referências}

ANDERSON, T. E.; ROBERTS, D. W. Compatibility of Beauveria bassiana Isolate with Insecticide Formulations Used in Colorado Potato Beethe (Coleotera: Crysomelidae) control. Journal of Economic Entomology, Annapolis Road, v.76, p.1437-1441, 1983.

BENZ, G. Environment. In. FUXA, R.; TANADA, Y. (Eds.). Epizootiology of insect diseases. New York: Wiley, 1987. p.177-214.

DUARTE, A.; MENENDEZ, J. M.; TRIGUEIRO, N. Estudio preliminar sobre la compatibilidad de Metarhizium anisopliae com algunos plaguicidas quimicos. Rev. Baracoa, La Habana, v.22, p.31-39, 1992.

GHINI, R.; KIMATI, H. Resistência de fungos a fungicidas. Jaguariuna: EMBRAPA/MA, 2000.

HALLEY, B. A. et al. (Eds.). Environmental effects of the usage of avermectins in livestock. Environmental impact of avermectin usage in livestock. Veterinary Parasitology, Amsterdam, v.48, p.109-125, 1993.

MOINO JUNIOR, A.; ALVES, S. B. Efeito de imidacloprid e fipronil sobre Beauveria bassiana (Bals.) Vuill. e Metarhizium anisopliae (Metsch.) Sorok. no Comportamento de limpeza de Heterotermes tenuis (Hagen). Anais da Sociedade Entomológica do. Brasil, Londrina, v.27, p.611-619, 1998.

NEVES, P. M .O. J. Compatibility of entomopathogenic fungi with neonicotinoid insecticides. Neotropical Entomology, Londrina, v.30, p.263-268, 2001.

TODOROVA, S. I. Compatibility of Beauveria bassiana with selected fungicides and herbicides. Environmental Entomology, Lanham, v.27, p.427-433, 1998. 